\title{
ArtigoOrigne
}

\section{AVALAÇAOO DA REDUÇÃO DE GRADIENTES PRESSÓRICOS E DA HIPERTROFIA VENTRICULAR APÓS VALVOPLASTIA CIRÚRGICA NA ESTENOSE AÓRTICA}

*G. R.Hoppen, R.A.K. Kall, F. B. Ludwg, A. D'Anla

Senviço de Cirurgia Cardiovascular; Instituto de Cardiologia do Rio $\mathrm{G}$ randedo Sul; FundaçãoUniversitária deCardiologia (IC/ FUC)

RESUMO - InTRODUção. A correção cirúrgica da estenose aórtica resulta em redução significativa do gradiente pressórico transvalvar, sendo acompanhada por regressão da hipertrofia ventricular esquerda(HVE). A intensidade ea rapidez dessa regressão tem sido objeto de avaliações. $A$ associação de valvoplastia aórtica e regressão imediata da HVE é relatada em poucos estudos.

Métodos. Foram estudados, prospectivamente, 11 pacientes submetidos à valvoplastia em estenose aórtica, utilizando-se ecocardiografia imediatamente antes da cirurgia e no período pós-operatório precoce $(6,1 \pm 0,9$ dias).

Resultados. A espessura septal variou de $12,10 \pm 1,66 \mathrm{~mm}$ para $11,36 \pm 1,12 \mathrm{~mm}$ (redução de 6,1\%) (NS) enquanto a espessura parietal variou $4,4 \%$ (de $11,70 \pm 1,41 \mathrm{~mm}$ para $11,18 \pm 1,16 \mathrm{~mm}$ ) (NS). A fração de ejeção apresentou uma variação de 62,02 \pm $18,59 \%$ para $62,50 \pm 11,74 \%$ (NS). A massa ventricular esquerda variou em $6,7 \%$ (de 277,65 $\pm 114,80$ g passou para $258,93 \pm 92,38 \mathrm{~g}$ ) (NS), 0 gradiente transvalvular médio regrediu de $53,6 \pm 10,3$

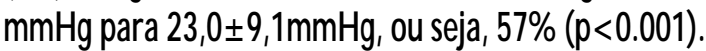

CONCLUSÖEs. A valvoplastia alivia 0 gradiente pressórico aórtico satiffatoriamente e a regressão da HVE tende a se iniciar logo após, porém ainda não é significativa no período pós-operatório imediato.

Unitermos: Estenose aórtica. Hipertrofia miocárdica. Valva aórtica.

\section{INTRODUÇão}

A hipertrofia ventricular esquerda (HVE), secundáriaàestenoseaórtica, éum mecanismo compensatório decorrentedo aumento de pressão e de volume intracavitários ${ }^{1}$. A correção cirúrgica da estenose aórticaresultaem redução significativa do gradiente pressórico transvalvar, sendo acompanhada por regressão, pelo menos parcial, daHVE ${ }^{1,2}$. A regressão daHVE após substituição da válvula aórtica é um parâmetro direto paraquantificar areduçãa do trabalho do ventrículo esquerdo eestáassociadaà redução dos riscos alongo prazo de insuficiência cardíaca congestiva e de morte súbitaassociados com aHVE ${ }^{1,3}$.

A ecocardiografia bidimensional é 0

*Correspondêndia:

Av. Princesa Isabel, 395 -Santana -Cep: $90620-001$ Porto Alegre/ RS-Tel.: (51)230-3600 r. 3557/ 3777 -E-mail: pesquisa@cardnettche.br método não-invasivo de maior acuráciana detecção e avaliação da severidade daHVE, além de estar sendo empregada na demonstraçãa da regressão da massa ventricular, apóscirurgiade correção deválvula aórticaestenótica, ${ }^{1,2}$.

Muitosestudostêm demonstrado aregressão daHVE apóstrocadeválvulaaórtica estenosada. Entretanto, não háconcordânciana literatura quanto ao período em que ocorre tal regressãa $0^{1-5}$. Estudos recentes mostram umasignificativaredução namassa ventricular esquerdanum tempo médio de 5 dias $(3,5-7,2)$ após substituição valvar, enquanto outros observaram esta modificação em tempo superior a 6 meses ${ }^{1,2,6}$.

A associação de valvoplastia cirúrgica aórtica e regressão da massa ventricular esquerdatêm sido alvo de poucosestudos. Acredita-se que, à semelhanç̧a das próteses, tal regressão sejalenta. Entretanto, não háavaliação desse problemadisponível na literatura. 0 objetivo deste estudo é avaliar o grau eavelocidade de regressão da massaventricular esquerda, através deecocardiografiabidimensional, apósvalvoplastia em válvula aórtica estenótica, no período pós-operatório imediato.

\section{Material e Métodos}

Para avaliar a regressão da hipertrofia ventricular esquerda foram estudados, prospectivamente, 11 pacientes submetidos à valvoplastia aórtica no período de janeiro a outubro de 1998 no Instituto de Cardiologia do Rio Grande do Sul, Fundação U niversitáriade Cardiologia. Foram incluídos no estudo apenas pacientes sintomáticos, com estenose aórtica moderadaa severa, com hipertrofiaventricular esquerda, sem outras lesões associadas, de etiologiacongênita ou senil, operados consecu- 

Figura 1 - Alterações no gradiente transvalvar máximo
(Grad Max) nosperíodos prée pós-operatório.

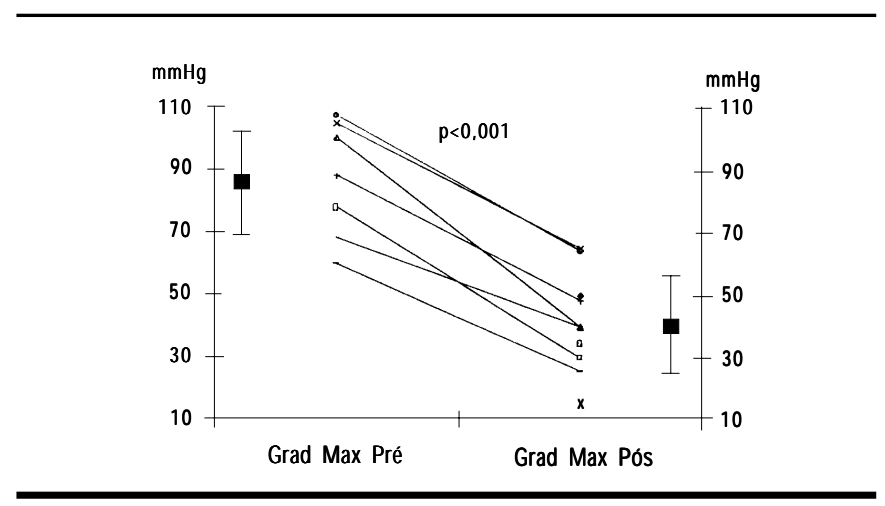

Figura 2 - Alterações no gradiente transvalvar médio (Grad Med) nosperíodos prée pós-operatório.

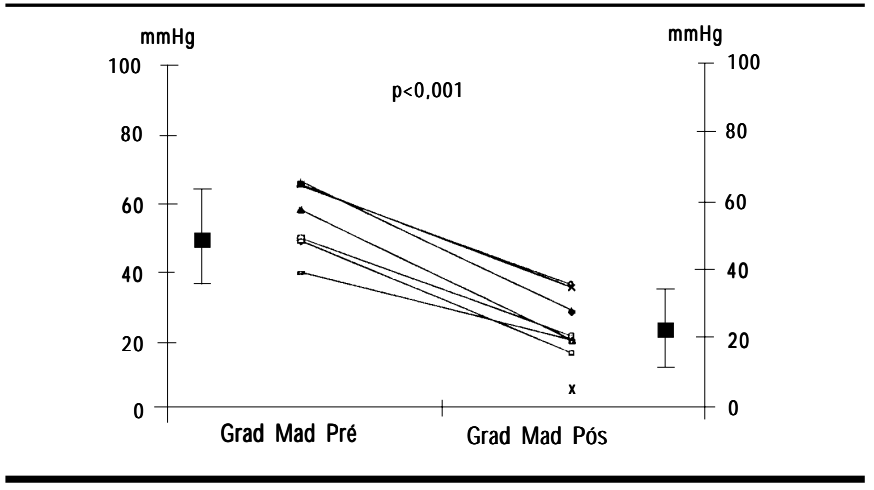

tivamente. Em todosos pacientesfoi realizadaecocardiografiabidimensional (mapeamento com fluxo a cores e D oppler) imediatamente antes da cirurgiae entre $05^{\circ} \mathrm{e}$ $07^{\circ}$ dias de pós-operatório, sendo avaliadas as seguintes medidas: espessuraseptal, espessurada parede ventricular esquerda posterior, fração de ejeção, massa ventricular esquerdae gradientes transvalvar máximo e médio. Todos os exames foram realizadospelo mesmo ecocardiografista. A análise dasimagens, bem como a obtenção dos dados, foram feitas em tempo real e analisadas por umúnico observador. Parao cálculo damassa ventricular esquerda, utilizou-se a seguinte equação, descrita por Devereuse et al.?:

$\operatorname{MVE}(g)=1,04\left([\text { LVID + PWT + IVST }]^{3}-\right.$ $\left.\operatorname{LVID}^{3}\right) \times 0,8+0,6$, onde:

$\mathrm{LVID}=$ dimensão internado ventrículo esquerdo;

PWT = espessuradaparede posterior;

IVST = espessurado septo intraventricular medido nadiástole final;

1,04 = gravidadeespecíficado miocárdio; $0,8=$ fator de correção.

Em todosos casos, a cirurgiafoi realizadaatravés detoracotomiamedianasob circulação extracorpóreaempregando-seoxigenadores de membrana, com hipotermia sistêmicamoderada $\left(\right.$ de $30^{\circ} \mathrm{a} 32^{\circ} \mathrm{C}$ ) e para- da isquêmica do coração sob cardio plegia hipotérmica cristalóide infundida por via anterógrada, drenando-se as cavidadesesquerdas através de aspirador em átrio esquerdo. A valva aórtica foi abordada por aortotomia oblíquae as calcificações foram retiradas mediante pinçassaca-cálcio, auxiliadas por aspiração e raspagem das válvulas. A descalcificação foi seguida por comissurotomiaquando necessário ${ }^{8}$.

No período do estudo foi realizado valvoplastiaaórticaem 11 pacientes, sendo $6(54,5 \%)$ do sexo masculino e $5(45,4 \%)$ do sexo feminino, com idade média de $68,72 \pm 9,98$ anos, variando entre 50 e 79 anos.

0 tempo médio entre a cirurgia e a realização daecocardiografiano pós-operatório foi de 6,09 $\pm 0,9$ dias.

Todos os dados foram coletados de forma prospectivae sofreram análise multivariada através de programa estatístico EPI-INFO, para computador, considerando-se umalfacrítico de $5 \%$ eexpressandose os valores sob a forma de média \pm desvio-padrão ( $m \pm D P)$.

\section{Resultados}

N ão houveóbitos durante afase hospitalar. Ao analisarmoso gradiente transvalvar máximo, notamosque ocorreuumaredução de $52 \%$ no período pós-operatório, regredindo de $86,53 \pm 18,5 \mathrm{mmHg}$ para $41,47 \pm 15,7 \mathrm{mmHg}(p<0.001)$. Em relação ao gradiente transvalvar médio, notamos que ocorreu uma redução de $53,56 \pm 10,3 \mathrm{mmHg}$ do pré-operatório para 23,0 $\pm 9,1 \mathrm{mmH}$ g no pós-operatório $(p<0,001)$, o que representauma redução de $57 \%$. (Fig. 1 e2). A espessura septal no período pré-operatório foi $12,1 \pm 1,66 \mathrm{~mm}$ eno pós-operatório esse valor mudou para $11,36 \pm 1,12 \mathrm{~mm}$ (N S), representando uma redução médiade 6,1\% (Fig. 3).A espessura parietal mudou de $11,70 \pm 1,41 \mathrm{~mm}$ no pré-operatório para $11,18 \pm 1,16 \mathrm{~mm}$ no pós-operatório (N S), o que representauma redução médiade 4,4\% (Fig. 4).

A fração de ejeção foi de $62,02 \pm$ $18,59 \%$ no pré-operatório e de $62,5 \pm$ $11,74 \%$ no pósoperatório, como podeser visto nafigura5, não expressando variação significativa dos pontos de vista clínico e estatístico desteparâmetro no período pósoperatório imediato.

Em relação à massa ventricular esquerda, notamos que houve uma tendência a redução estimadaem $6,7 \%$ no pós-operatório em relação ao período pré-operatório (Fig. 6), ou seja, era de 277,65 $\pm 114,8 \mathrm{ge}$ mudou para $258,93 \pm 92,38 \mathrm{~g}$ (N S). 
Figura 3 - Alterações na espessura (Esp) septal nos períodos prée pós-operatório.

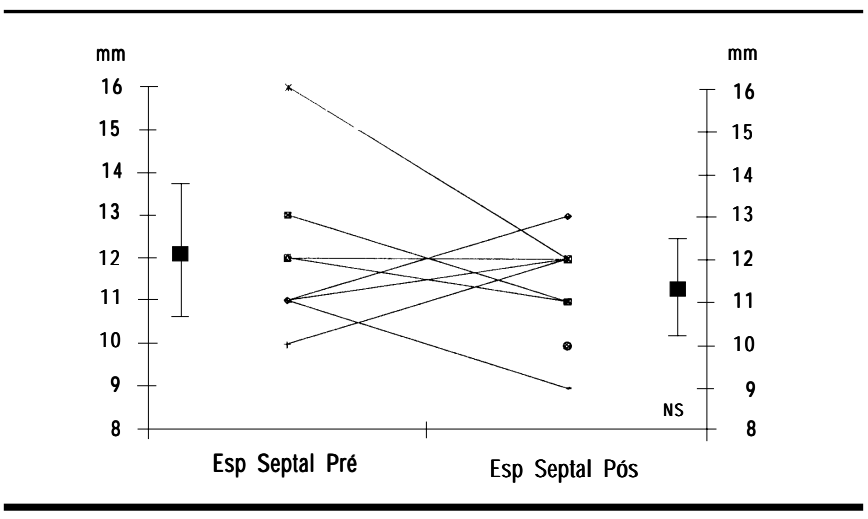

Figura 4 - Alterações na espessura (Esp) parietal nos períodos préepós-operatório.

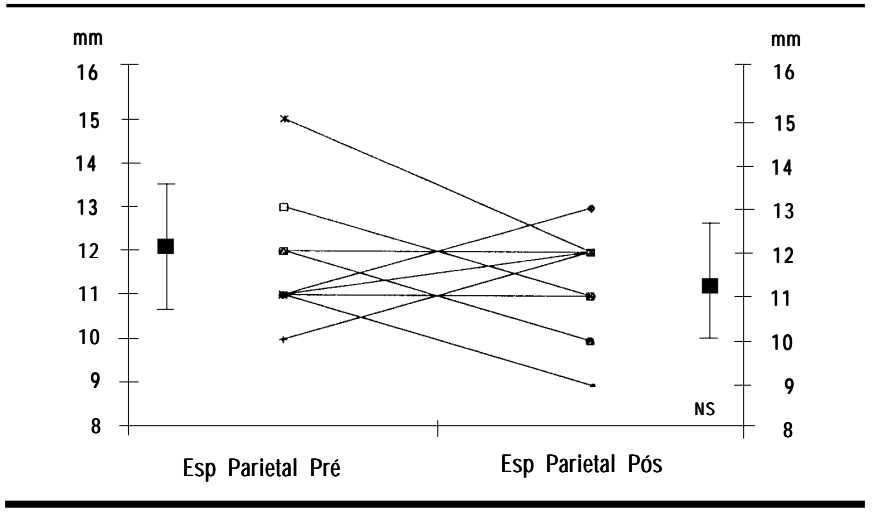

Entre os pacientes analisadosneste estudo, não ocorreu nenhum caso de insuficiência aórtica após a realização da valvoplastiacirúrgica.

\section{Discussão}

Durante as décadas de 1950 e 1960, debridamento e comissurotomia eram os únicosprocedimentos disponíveis paraaliviar aestenose da válvula aórtica?. Com 0 advento das próteses valvares, biológicas ou mecânicas, estesprocedimentoscaíram em desuso. Como passar dosanos, notouse que as próteses valvares têm alguns inconvenientes, tais como a existência de gradienteselevadosnasprótesesbiológicas menores, anecessidade de anticoagulação nas próteses metálicas, e 0 aumento no risco de tromboembolismo e de complicações infecciosas ${ }^{10,11}$. 0 interesse napreservação daválvulaaórticanativaressurgiu com a intenção de evitar essas complicações, facilitar o procedimento em pacientes com anel aórtico pequeno, os quais necessitariam de ampliação do anel aórtico, e obter um resultado paliativo satisfatório em pacientesidosos ${ }^{10}$.

A hipertrofia do ventrículo esquerdo, devido à sobrecargade volume ou depressão, é uma adaptação para compensar as altas pressõesintracavitárias ${ }^{1,2}$. Moderada ou severahipertrofiaventricular esquerda muitas vezeséa causa de arritmias, insuficiênciacardíaca congestiva ou de morte súbita ${ }^{3}$. A correção cirúrgicadaestenose aórtica resultaem redução significativado gradiente transvalvar, sendo acompanhada por regressão, pelo menos parcial, da HVE ${ }^{1,2}$. Muitosestudostêm demonstrado aregressão da HVE após troca de válvula aórtica estenosada. Entretanto, não háconcordânciana literaturaquanto ao período em que ocorre tal regressão ${ }^{1-5}$. Estudos recentes mostram umasignificativaredução namassa ventricular esquerdanum tempo médio de 5 dias (3,5-7,2) após substituição valvar, enquanto outrosobservaram estamodificação em tempo superior a 6 meses ${ }^{1,2}$.

0 grau de retorno ao normal da massa ventricular esquerdaapósanormalização da pressão sistólicaventricular por valvoplastia cirúrgicaou por substituição protéticadependedaextensão dasalterações degenerativas miocárdicas que jáhouveram ocorrido eda grandezadahipertrofiajádesenvolvida, bem como da perda relativa da reserva ventricular ${ }^{12}$. A regressão continua por mais de um ano, mas normalização da massa ventricular raramenteéconseguida?2.

A massaventricular esquerdareflete a severidade da estenose aórtica e tem sido usadaem estudos, experimentaiseclínicos, parademonstrar aregressão dahipertrofia ventricular após a substituição da válvula aórtica'. No nosso estudo, embora não significativa, manifestou-seumatendênciaa redução média de $6,7 \%$ na massa ventricular esquerdaem $6,09 \pm 0,9$ dias de pósoperatório de valvoplastiaaórtica. Emestudo realizado por Christakis et al. ${ }^{1}$ foi demonstrado redução de $10 \%$ na massa ventricular esquerdaem umamédiade 4,9 diasapós substituição da válvula aórtica.

Sabe-se que no período precoce, após atrocavalvar, existe edemasubstancial nos tecidos, especialmente no tecido miocárdico, e isto pode alterar as medidas das paredes do ventrículo esquerdo ${ }^{1}$. Estasalteraçõespodem influir nas medidas ecocardiográficas, atenuando a diminuição esperada das espessuras septal e parietal. N esta série, ao tempo dosexames, jáseesperaria resolução do edema, porém não hácerteza nem método adequado paraavaliação desse fenômeno. A presença de edema tecidual poderia explicar 0 aumento que ocorreu em alguns parâmetros, em casosiso lados, no período pós-operatório.

Em trabalho publicado por St. John Suttonet al. ${ }^{4}$ foi demonstrado melhorasignificativanafração de ejeção em umamédia de 6 semanas de pós-operatório de substi- 
Figura 5 - Alterações na fração de ejeção (FE) nos períodos prée pós-operatório.

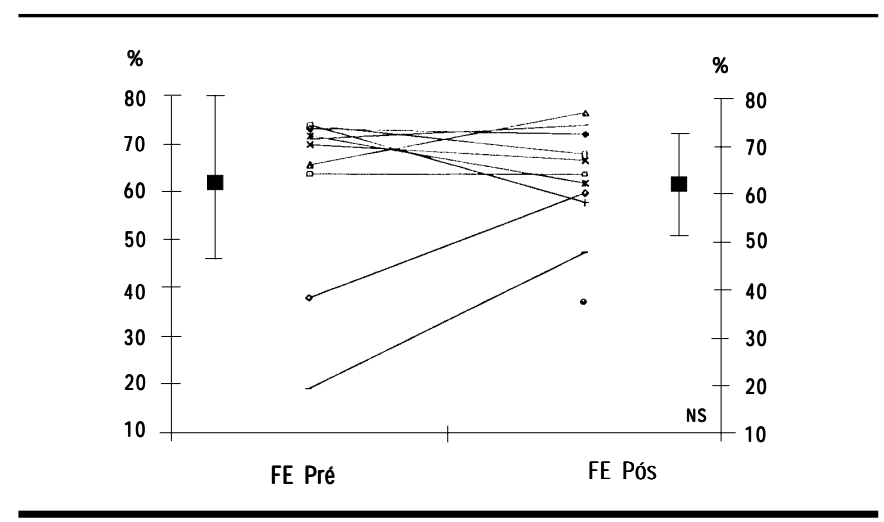

Figura 6 - Alterações na massa ventricular esquerda (MVE) nos períodospréepós-operatório.

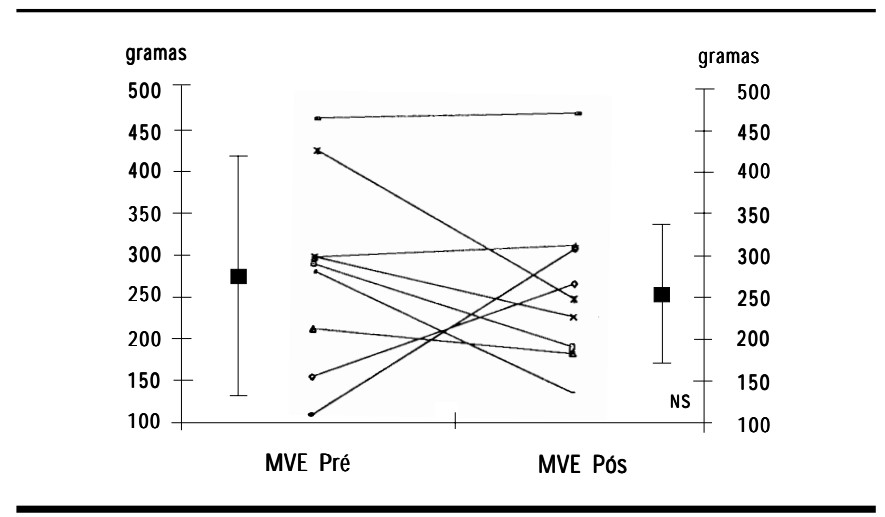

tuição de válvula aórtica. Estamelhorafoi mais expressivaparaaqueles pacientesque apresentavam fração deejeção em torno de $20 \%$ quando comparados com aqueles com fração de ejeção maior que $50 \%$. Em nosso estudo a média de fração de ejeçãa no pré-operatório foi de $62,02 \pm 18,59 \%$ (variando de $18,9 \%$ a $74 \%$ ) eno pós operatório esta médiafoi de $62,05 \pm 11,74 \%$ (variando de $37,3 \%$ a $76,9 \%$ ). Como pode ser visto na figura 5 os maiores aumentos ocorreram naqueles pacientes quetinham as frações de ejeção mais baixas no préoperatório.

$\mathrm{N}$ ascirurgias depacientes comestenose aórticae anel aórtico pequeno, para evitar cirurgiascommaior morbidade, taiscomo as de ampliação do anel aórtico, 0 cirurgião muitasvezesimplantaprótesesvalvaresmenores, as quais geram maiores gradientes pressóricostransvalvularesno pós-operatório e menor regressão da hipertrofia ventricular esquerda ${ }^{3.5}$. Nesses pacientes, a valvoplastiaaórticatorna-seuma alternativa com baixa morbidade, principalmente nos pacientesmaisidosos. N o nosso estudo observamosque avalvoplastiaaórticaocasionoureduçõesde $52 \%$ e $57 \%$ ( $p<0,001)$ nos gradientestransvalvar máximo emédio, respectivamente, no período pós-operatório. 0 gradientetransvalvar médio pós-operató- rio nesteestudo foide $23,0 \pm 9,1 \mathrm{mmHg}$. Em trabalho publicado por Gonzáles-Juanatey et al. ${ }^{5}$, em pacientes usando próteses valvaresaórticas (biológicasemetálicas) de 19 $\mathrm{mm}, 0$ gradiente transvalvar médio foi de $18,3 \pm 7 \mathrm{mmH} \mathrm{g}$. Em outrapublicaçãa $0^{3} 0$ gradientetransvalvar médio pós-operatório foi de $18,2 \pm 4,6 \mathrm{mmH}$ g ao seutilizar próteses biológicas de $21,4 \pm 0,9 \mathrm{mme}$, ao se utilizar prótesesmetálicas demesmo tamanho, esse gradientefoide $15,7 \pm 3,8 \mathrm{mmHg}$.

Podemos concluir que a valvoplastia paraestenose aórticareduz adequadamente o gradiente pressórico transvalvar e a regressão dahipertrofiaventricular esquerdase inicialogo apósavalvoplastiaaórtica, emboranão sejasignificativanos períodos maisprecocesde pós-operatório. 0 utros estudos devem ser realizadosem períodos mais tardios de pós-operatório de valvoplastiaaórticapara comparar aregressão da hipertrofiaventricular esquerdacom aquela que ocorre, conforme outros relatos da literatura, após a substituição da válvula aórticapor prótesesmecânicasebiológicas.

\section{SummarY}

Left Ventricular Hypertrophy Regression Immediately After Aortic Valve Repair.
BackGRound. Relief of gradient is followed by myocardial mass reduction in aortic stenosis. Its degree and speed are under evaluation. Aortic valve repair in calcified aortic stenosis is less well studied than replacement.

Methods. We evaluated left ventricular hypertrophy reduction by echocardiogram in 11 patients immediately after valve repair in aortic stenosisat a mean of $6.1 \pm 0.9$ days post operative.

Results.Septal width was $12.10 \pm 1.66$ $\mathrm{mm}$ pre and $11.36 \pm 1.12 \mathrm{~mm}$ post operative, 6,1\% reduction (NS). Parietal width varied $4.4 \%$ from $11.70 \pm 1.41 \mathrm{~mm}$ to $11.18 \pm 1,16 \mathrm{~mm}$ (NS). Ejection fraction went from $62.02 \pm 18.59 \%$ to $62.50 \pm 11$. $74 \%$ (NS). Left ventricular mass varied $6.7 \%$, from $277.65 \pm 114.80 \mathrm{~g}$ to $258.93 \pm$ $92.38 \mathrm{~g}$ (NS). Mean transvaluar gradient reduced $57 \%$, from $53.56 \pm 10.30$ to $23.0 \pm 9.1 \mathrm{mmH} \mathrm{g}(P<0.001)$.

Conclusion. Aortic valve repair reduces gradients adequately and left ventricular hypertrophy shows a trend to regression soon after aortic repair, but is not yet significant in the first post-operatively week. [Rev Ass Med Bras; 46(4): 354-8]

Key Words: Aortic Stenosis. Myocardial hypertrophy. Aortic valverepair. 


\section{Referências Bibliográficas}

1. Christakis $G T$, Joyner $C D, M$ organ $C D$, et al. Left ventricular mass regression early after aortic valve replacement. Ann Thorac Surg 1996; 62: 11084-89.

2. Panidis JP, Kotler MN, Ren JF, et al. Development and regresion of left ventricular hypertrophy. J Am Coll Cardiol 1984; 3: 1309-20.

3. De Paulis R, Sommariva L, Colagrande L, et al. Regression of left ventricular hypertrophy after aortic valve replacement for aortic stenosis with different valve substitutes. J Thorac Cardiovasc Surg 1998; 116: 590-98.

4. St. John Sutton M, Plappert T, Spiegel A, et al. Early postoperative changes in left ventricular chamber size, architecture and function in aortic stenosis and aortic regurgitation and their relation to intrao perative changes in after- load: a prospective two-dimensional ecocardiographic study. Circulation 1987; 76:77-89.

5. Gonzalez-JuanateyJR, García-A cuñajM, Fernandez MV, et al. Influence of the size of aortic valve prostheses on hemodynamics and change in left ventricular mass: implications for the surgical management of aortic stenosis. J Thorac Cardiovasc Surg 1996; 112:273-80.

6. Maselli D, Pizio R, Bruno LP, Di Bella I, De $\mathrm{G}$ asperis $C$. Left ventricular mass reduction after aortic valve replacement: homografts, stentless and stented valves. Ann Thorac Surg. 1999; 67:966-71.

7. O h JK, Seward JB, Tajik AJ. Ecocardiografia, Clínica Mayo - Avaliação dafunção ventricular. Rio de Janeiro: M edsi, 1997:42-43.

8. Kalil RAK, TeixeiraFilho GF, Sant'AnnaJRM, et al. Recuperação por debridamento manual da valva aórtica estenótica calcificada. RevBras Cir
Cardiovasc 1995;10(2):65-69.

9. Shapira N, Lemole GM, Fernandez J, et al. Aortic valve repair for aortic stenosis in adults. Ann Thorac Surg 1990; 50:110-20.

10. BaezaO R, Majid N K, Conroy DP, et al. Combined conventional mechanical and ultrasonic debridement for aortic valvular stenosis. Ann Thorac Surg 1992; 54:62-7.

11. Mindich BP, Guarino T, Goldman ME. Aortic valvulo plasty for acquired aortic stenosis. Circulation 1986; 74:130-35.

12. KennedyJW, Doces], Stew art D K. Left ventricular function before and following aortic valve replacement. Circulation 1977; 56:944-50.

Artigo recebido: 15/12/99

Aceito para publicação: 26/06/00 